Instruments and Methods

\title{
Snow replica method for three-dimensional X-ray microtomographic imaging
}

\author{
Martin HEGGLI, Esther FREI, Martin SCHNEEBELI \\ WSL Institute for Snow and Avalanche Research SLF, Flüelastrasse 11, CH-7260 Davos-Dorf, Switzerland \\ E-mail: heggli@slf.ch
}

\begin{abstract}
Visualization and quantification of snow structures at a scale of a few millimetres is important in understanding the mechanical, thermal and electromagnetic properties of snow. Surface sections and, to an even greater degree, three-dimensional (3-D) reconstructions of cast snow samples are difficult to prepare, and automatic image processing is notoriously difficult and often requires manual evaluation. Here, we present a new method to measure the 3-D structure of cast snow samples. Snow samples cast with diethyl phthalate (DEP) and frozen are cut to a sample size a few centimetres in diameter and up to $10 \mathrm{~cm}$ in height. The ice of these samples is then sublimated in high vacuum and the remaining negative structure (replica) is imaged using X-ray microtomography (micro-CT). The accuracy of the method is demonstrated by comparing micro-CT scans of the original snow structure and the replica. The method described here allows easy transportation of samples, requires little manual interaction, has a very high spatial resolution of up to $10 \mu \mathrm{m}$ and is environmentally friendly.
\end{abstract}

\section{INTRODUCTION}

Snow is a sintered porous material consisting of a continuous ice network and a continuous pore space in between. The direct observation of snow stratigraphy and grain size in a snow pit is limited to a resolution of about $1 \mathrm{~cm}$. Two-dimensional surface sections can be carried out on larger samples (Pielmeier and Schneebeli, 2003; Matzl and Schneebeli, 2006). The microstructure of snow determines many properties such as mechanical strength, thermal conductivity, surface chemistry and albedo. It is hence important to characterize snow microstructure on the submillimetre level. It is crucial to know the full threedimensional (3-D) microstructure of snow, especially for numerical simulations (Schneebeli, 2004; Kaempfer and others, 2005; Kaempfer and Schneebeli, 2007).

There are two methods for characterizing the microstructure of snow: serial sectioning (Perla and others, 1986; Good, 1987; Schneebeli, 2001) and micro-computer tomography (micro-CT) (Brzoska and others, 1999; Coléou and others, 2001; Flin and others, 2003; Schneebeli, 2004; Schneebeli and Sokratov, 2004).

Micro-CT is a powerful method for the analysis of microstructures, which has been applied in many areas such as materials science, geosciences, biology and archaeology (see, e.g., Stock, 2008). It reveals microstructural details with a spatial resolution of $5-80 \mu \mathrm{m}$, and its application is relatively straightforward and time-efficient. Micro-CT has been demonstrated to be applicable to snow (Flin and others, 2003; Schneebeli and Sokratov, 2004). Kerbrat and others (2008) showed that micro-CT has a sufficient resolution to resolve the fine details of snow, even for most types of new snow. However, the application of this method was restricted to snow sampled in locations close to the laboratory where the micro-CT instrument is operated because long storage or transport of samples leads to changes in the snow microstructure due to metamorphism and the fragility of snow. Therefore, samples that need to be transported to a suitable laboratory from remote locations, such as Greenland or Antarctica, or that cannot be processed immediately must be conserved by casting.

Two substances have been used for casting and conserving snow samples. Firstly, 1-chloronaphthalene permits direct measurements of cast samples in the micro-CT instrument (Flin and others, 2003). However, this substance is hazardous to both humans and the environment and has an unpleasant odour (Howe and others, 2001). Diethyl phthalate (DEP) or dimethyl phthalate, on the other hand, is less hazardous and almost odourless (Sekizawa and Dobson, 2003).

In principle, reconstruction of the 3-D microstructure of snow from serial sections of DEP cast samples is straightforward. However, in practice, analysing the images is difficult because DEP tends to recrystallize. This results in a very similar appearance of ice and DEP in the image and makes image segmentation difficult, if not impossible. Thus far, micro-CT could not be used to study DEP-cast samples because the X-ray attenuation coefficients of DEP and ice are almost identical, thus making it impossible to detect any contrast in an X-ray tomogram. The X-ray attenuation coefficient of 1-chloronaphthalene, $10-30 \mathrm{keV}$, is about three times that of ice. Therefore, an absorption contrast between 1-chloronaphthalene and ice can be observed. However, the typically unavoidable presence of air inclusions in any cast sample requires sophisticated algorithms for correct image segmentation because the CT image of a three-phase material is inherently more difficult to segment.

Here, we present a new method that allows the investigation of the microstructure of snow samples cast in DEP with micro-CT despite the missing absorption contrast between the two substances. Samples are collected and cast with DEP. In this form, samples can be transported and stored. Before the micro-CT measurement, the ice is sublimated out of the cast samples. This is possible because between 250 and $270 \mathrm{~K}$ the vapour pressure of ice is about $100 \mathrm{~Pa}$ (Wagner and others, 1994), whereas the vapour pressure of DEP in that range is about $10^{-4} \mathrm{~Pa}$ (Roháč and 


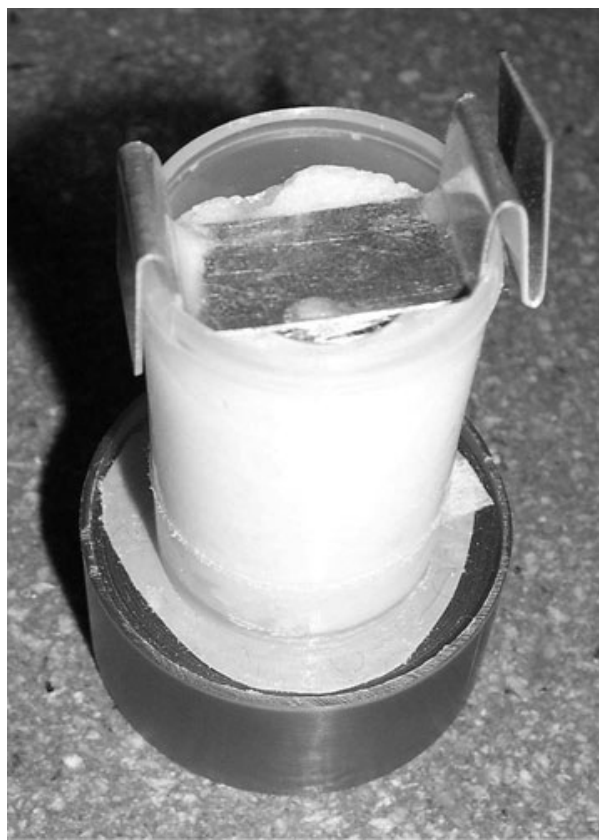

Fig. 1. Sample holder used for verification of micro-CT measurements. This sample holder was used to make micro-CT scans of the original snow sample, then cast the sample with DEP, perform the sublimation and finally acquire a second micro-CT scan of the replica. The aluminium strip serves to prevent the snow sample from rotating or floating on the DEP. Note that DEP $\left(1130 \mathrm{~kg} \mathrm{~m}^{-3}\right)$ is denser than ice $\left(917 \mathrm{~kg} \mathrm{~m}^{-3}\right)$.

others, 2004). (The values at $253 \mathrm{~K}$ are $101 \mathrm{~Pa}$ for ice and $1.36 \times 10^{-4} \mathrm{~Pa}$ for DEP, and at $268 \mathrm{~K}$ are $396 \mathrm{~Pa}$ for ice and $2.15 \times 10^{-3} \mathrm{~Pa}$ for DEP.) This enormous difference (almost six orders of magnitude) in the vapour pressures has the effect that only the ice sublimates. Under normal freezer conditions, this sublimation process takes several years. To accelerate it we prepared the samples at a pressure of about $1 \mathrm{~Pa}$, i.e. two orders of magnitude below the vapour pressure of ice at $-20^{\circ} \mathrm{C}$ but four orders of magnitude above the vapour pressure of DEP at that temperature.

When all the ice has sublimated, the remaining DEP structure represents a negative of the original snow structure. It is then straightforward to acquire a micro-CT scan of the negative DEP structure. After image segmentation the inverse of the DEP structure represents a digital replica of the original snow microstructure. The data can be used in exactly the same way as micro-CT measurements acquired directly from scanning a snow sample (e.g. to calculate parameters such as density or specific surface area (SSA) (Kerbrat and others, 2008), perform numerical simulations (Schneebeli, 2004; Schneebeli and Sokratov, 2004; Kaempfer and Schneebeli, 2007) or visualize the 3-D microstructure of the sample (Greene, 2007)).

In this study, we evaluate the feasibility of the suggested method including the time required to complete the sublimation and the accuracy with which the original snow microstructure can be reproduced. For this purpose, different snow samples were scanned using micro-CT. Subsequently, these samples were cast with DEP and the ice was removed by sublimation. After a constant sample weight had been reached, the samples were re-scanned and the resulting images compared with those of the original snow. Furthermore, structural parameters calculated on the basis of the digital replica were compared with those calculated directly from the snow.

\section{MATERIALS AND METHODS}

Two different types of samples were used in the experiments described here. First, to verify the method, we prepared snow samples directly in CT sample holders. CT scans of the original snow structure were measured from these samples to identify the initial snow structure. Subsequently, this snow was cast with DEP as described below to fill the pore space completely. The ice was then removed from these samples by sublimation under vacuum. A second CT scan was acquired after all ice had been removed from the sample. This allows an effective comparison between the replica and the original snow structure and, therefore, tests the accuracy of the proposed method.

Second, for future application of our method the snow samples will be cast with DEP directly in the field. To test the processing of such field samples, snow was cast with DEP in blocks of $\sim 70 \mathrm{~mm} \times 70 \mathrm{~mm} \times 70 \mathrm{~mm}$. From the cast blocks, cylindrical samples were prepared that fit well in standard CT sample holders with a diameter of $20 \mathrm{~mm}$.

\section{Sample preparation}

\section{Phthalate casting}

For verification purposes, some samples were prepared with CT scans taken at the beginning and the end of the experiment. A snow sample was put in a small polyethylene beaker that was mounted with double-sided adhesive tape on the base part of a cut $36 \mathrm{~mm}$ sample holder (Fig. 1). An aluminium strip with a pushpin with three spikes fixed at the bottom held the snow down to prevent it from floating on the liquid DEP. Additionally, two thin holes were made at both sides of the sample. The holes facilitate the filling of the pore space with DEP because they allow the filling process to start from the bottom of the beaker. Care had been taken not to disturb the structure of the remaining snow.

A first CT scan of the original snow structure was taken at this stage. The sample was then cast with diethyl phthalate (purum, Fluka, Sigma-Aldrich AG, Buchs, Switzerland). Both the snow sample and the DEP were cooled to $-5^{\circ} \mathrm{C}$. The casting was undertaken in a cold room at $-5^{\circ} \mathrm{C}$. After cooling with dry ice for about 2 hours the DEP had solidified. A second CT scan was taken of the same sample region to make sure that the casting had completely filled all pores of the snow microstructure. If air bubbles are found in this scan, these images can be used to correct the final images. Samples were then stored under vacuum as described below to sublimate all of the ice from the sample. After constant sample weight had been reached, a final CT scan was acquired.

\section{Sample trimming}

In future applications, the preparation of snow samples will be effected through sampling and casting directly in the field. A detailed description of this method can be found in an online manual (M. Schneebeli and V. Smith, http:// www.slf.ch/forschung_entwicklung/schnee/schneestruktur/ projekte/replikamethode/Casting_Snow_Phthalate.pdf). The cast samples are transported to the laboratory where they will be processed further. To test the processing of such field samples, some similar samples were prepared in the laboratory. 
Sample trimming had to be conducted in a cold room at $-20^{\circ} \mathrm{C}$ to prevent the DEP from melting. The cast blocks with dimensions of $\sim 70 \mathrm{~mm} \times 70 \mathrm{~mm} \times 70 \mathrm{~mm}$ were first cut into quadratic prisms of $\sim 30 \mathrm{~mm} \times 30 \mathrm{~mm}$ using either a manual saw or a bandsaw. A mitre gauge was used with the manual saw to ensure orthogonal cuts. Care was taken to remove all marginal parts of the cast sample. Subsequently, cylindrical samples with a diameter of $18 \mathrm{~mm}$ and a length of $\sim 60 \mathrm{~mm}$ were turned on a lathe. Appropriate protective equipment (goggles, breathing masks, rubber gloves, lab coat) must always be worn while preparing the samples.

\section{Experimental set-up}

The cast samples were stored in a vacuum desiccator to sublimate the ice. The ground flange of the desiccator was lubricated (High Vacuum Grease, Dow Corning). The desiccator was continuously evacuated by a vacuum pump (TRIVAC DSE T1, Leybold). This high-capacity pump can reach an ultimate pressure of $0.2 \mathrm{~Pa}$ and has a volume flow rate of $5 \mathrm{~m}^{3} \mathrm{~h}^{-1}$. The pump was installed outside the cold room because of its substantial heat production. Rubber tubing was used to connect the pump with the desiccator inside the cold room. A Pirani cell was connected with the pump to measure the pressure in the vacuum system.

The samples were weighed before and after casting the snow samples with DEP as well as at regular intervals during the vacuum sublimation. Samples were stored under vacuum until their weight had reached a constant level. This was considered an indication that all the ice had sublimated out of the sample. At this point, the samples were ready to be (re-)scanned by micro-CT.

With some samples we tested the additional use of anhydrous calcium sulphate (Sikkon Fluka, Fluka AG, Buchs, Switzerland) as a drying agent. It was dried for a couple of hours in a drying oven at $240^{\circ} \mathrm{C}$ immediately before use, cooled to $-20^{\circ} \mathrm{C}$ in an airtight container and then put in the desiccator during the sublimation process.

\section{Computer tomography and analysis}

\section{Micro-CT measurements}

Micro-CT measurements were taken on a $\mu \mathrm{CT} 80$ (Scanco Medical AG, Brüttisellen, Switzerland) bench-top CT system that operates in cone-beam geometry. The micro-CT instrument was operated in a cold room at $-20^{\circ} \mathrm{C}$. The voxel size of the acquired images was either 10 or $18 \mu \mathrm{m}$. Turned samples were measured in a $20 \mathrm{~mm}$ diameter sample holder that allowed a voxel size of $10 \mu \mathrm{m}$. Verification samples, on the other hand, were prepared in a small polyethylene beaker that was mounted with double-sided adhesive tape on the ground part of a cut $36 \mathrm{~mm}$ sample holder (Fig. 1). These samples had to be measured with a voxel size of $18 \mu \mathrm{m}$. CT measurements were taken at a peak voltage of $55 \mathrm{kVp}$ and an intensity of $145 \mathrm{~mA}$. The exposure time was $400 \mathrm{~ms}$ without averaging. A total of 1000 projections was used for the reconstruction. Scanning a sample $5.6 \mathrm{~mm}$ in height took $\sim 1$ hour.

\section{CT data processing}

The CT data were processed and evaluated using IPL (Image Processing Language, Scanco Medical AG, Brüttisellen, Switzerland) supplied with the micro-CT instrument. Four IPL scripts were used for the different evaluation steps.

After reconstruction, the raw greyscale images were first filtered using a 3-D Gaussian filter ( $\sigma=1$ voxel, kernel half-

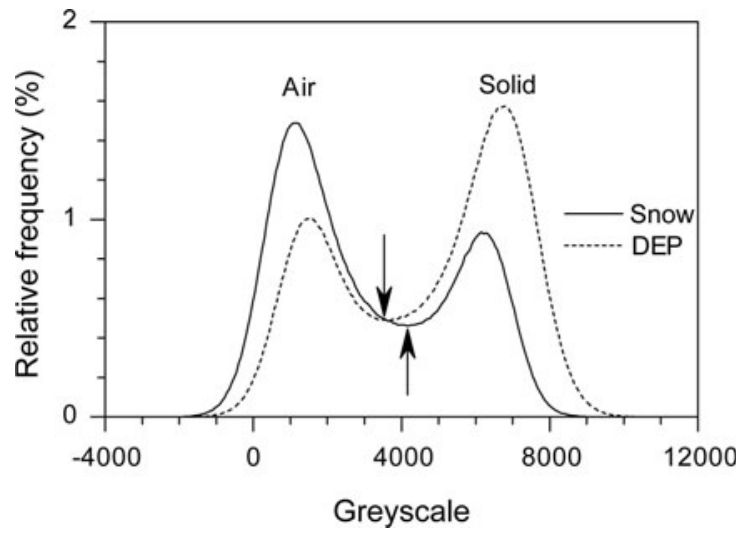

Fig. 2. Grey-level histograms of the filtered micro-CT images. The arrows indicate the local minima between the two peaks. The corresponding grey-level values have been used for image segmentation. The threshold values for the original snow sample and for the DEP replica differ slightly. They are 4247 for the threshold between air and ice (snow) and 3624 for air-DEP (replica).

width $=2$ voxels) to reduce noise in the images (Yoo and others, 2004). The optimal threshold value for image segmentation is based on the histograms of the greyscale values of the Gauss-filtered images (Fig. 2).

The threshold for image segmentation was determined as the local minimum between the two peaks in the histogram. The two peaks were well separated and the position of the minimum was easy to determine. The average of the minimum values of several volumes of interest (VOIs) in the samples was used as the threshold value for segmentation. The threshold used for segmentation of the snow samples differs from that for the replica. This is due to the slightly different X-ray attenuation coefficients of ice and DEP as well as to the different volume fractions of the solid phase. Therefore, the threshold values have to be re-determined for each snow type. The average threshold for the ice-air segmentation in our examples was at a grey value of 4247, whereas for DEP-air it was at 3624 (arrows in Fig. 2).

The segmented images were then subjected to a 3-D component-labelling procedure. In this, the individual disconnected objects in the volume were ordered according to descending volume. Only the largest connected component of the structure was retained and all others rejected, because they were considered as noise. The largest component in all cases contained $>99 \%$ of all structure voxels.

Subsequently, the snow images could be used, whereas the segmented images of the phthalate structure were inverted, i.e. after inversion all structure voxels were considered as pore space and vice versa. The inverted images were again component-labelled to remove unconnected parts. Small positioning errors between the different CT scans were taken into account: with an in-house Interactive Data Language (IDL) program the shift that minimizes the remaining differences between the two 3-D images was determined. The thickness of the structures in the difference volume was checked by eroding with the smallest possible structuring element.

For assessing the accuracy of the method, the 3-D difference image between the two scans was calculated by subtracting the segmented and inverted image of the replica 


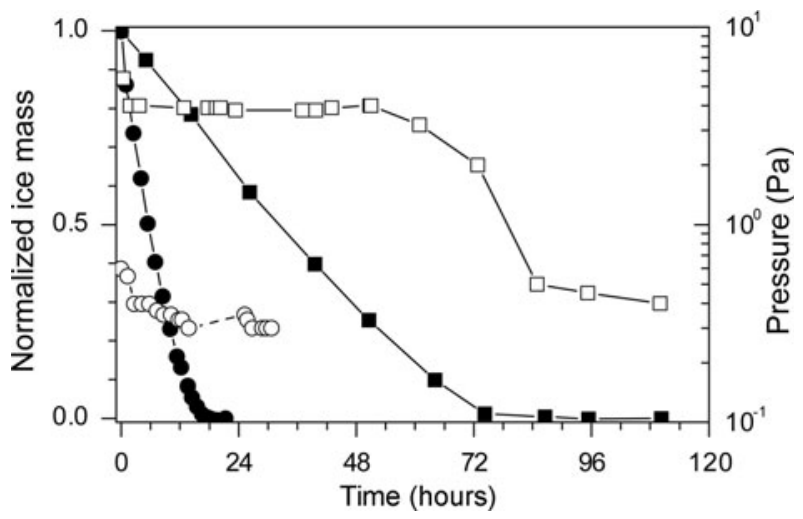

Fig. 3. Weight loss (solid symbols) and pressure (open symbols) during the vacuum treatment of two samples. Sample A (squares) was kept in the vacuum without drying agent; sample B (circles) was stored in the vacuum together with a drying agent. The connecting lines serve as a visual guide.

from the segmented image of the original snow. Furthermore, several structural parameters were calculated with IPL. The values obtained from the replica were compared with those from the original snow structure. Calculations were not based on the whole scanned sample volume but rather on a number of arbitrarily chosen non-overlapping sub-volumes with a size of $200^{3}$ voxels, i.e. $(3.6 \mathrm{~mm})^{3}$. The density was determined based on voxel counting in the segmented images, the specific surface area per ice volume (SSA) was calculated based on a surface triangulation method (Hildebrand and others, 1999), and the ice and pore thicknesses were determined by the distance transformation method (Hildebrand and Rüegsegger, 1997). To estimate the ice thickness, each point in the ice matrix was assigned a local thickness based on the diameter of the largest sphere containing that point and lying completely inside the ice structure. From these local thicknesses the thickness distribution was calculated and a volumeweighted mean thickness was determined from the arithmetic mean of the local thicknesses. The same procedure was applied to the pore space, yielding a value describing the pore width or the spacing between the ice structures. A discrete implementation of this method had to be used because of the discrete nature of the digital images.

\section{RESULTS}

\section{Vacuum pressure and weight loss of samples}

Sublimation of ice from the samples was followed by examination of their weight loss. The vacuum pressure also gives a good indication on the progress of the sublimation process. The net weight of the snow was determined for eight samples before casting them with DEP. This made it possible to check whether the snow had completely sublimated when the total sample weight did not change further. For all these samples it was observed that the snow weight decreased to zero, i.e. the snow was completely removed from the sample by the sublimation.

As a control, a sample of pure DEP was kept in the same vacuum as the samples for sublimation. For more than 1 month the weight of this DEP sample remained constant (weight change $<0.2 \%$ ). No sublimation could be measured at a pressure of $0.2 \mathrm{~Pa}$.
In Figure 3 we present the weight loss of two samples. In addition, the evolution of the vacuum pressure in the two experiments is shown. Sample A was in the desiccator as a single sample without a drying agent. When the weight had reached a constant value, the sublimation process was considered terminated, i.e. all ice had sublimated out of the sample. In this case, $5.3 \mathrm{~g}$ of ice could be removed from the sample. Completion of the process took $\sim 75$ hours (i.e. $\sim 3$ days). The pressure curve showed a more or less constant value of $\sim 4 \mathrm{~Pa}$ during sublimation. A considerable drop in the pressure to $\sim 0.4 \mathrm{~Pa}$ was observed when sublimation ended. When six samples were in the desiccator simultaneously, the sublimation process took $\sim 171$ hours (i.e. $\sim 7.5$ days), about twice as long as for a single sample.

Sample B was in the desiccator as a single sample with a drying agent (calcium sulphate). Instead of 75 hours, the sublimation process took only about 16 hours to complete. A similar acceleration was observed for two other samples. The drying agent reduced the time required by approximately a factor of five. The vacuum pressure curve also changed. From the beginning of the sublimation process, the vacuum pressure reached a value of $\sim 0.4 \mathrm{~Pa}$, i.e. the minimum value observed at the end of sublimation without a drying agent. In this experiment a drop in the pressure to about $0.3 \mathrm{~Pa}$ was observed after completion of the sublimation process. The drying agent accelerated sublimation appreciably, because it seems to effectively absorb the sublimated water molecules from the desiccator volume, thus lowering the water-vapour partial pressure in the desiccator.

For one sample, which was stored under vacuum without drying agent, the progress of a sublimation was followed in a series of micro-CT measurements. Scans were taken at the beginning, after 4 hours, after 12 hours and then at regular intervals of 12 hours (Fig. 4). The sublimation front progressed concentrically inward starting from the perimeter of the sample. It seems that ice is almost completely removed at a certain radius before the sublimation front progresses to a smaller radius. Ice structures were exposed all over the surface of the sample because the sample was turned on a lathe. The ice structure forms a continuous network. After sublimation of the ice, network forms escape channels for more sublimating water molecules. After $\sim 60$ hours the sublimation front had reached the centre of the sample. At this point, the weight had decreased to $10 \%$ of the initial value. During the following few hours only a residual weight loss of about $0.5 \mathrm{~g}$ was observed. The corresponding CT scans at 60 and 84 hours look almost identical. We can therefore assume that this residual weight loss originated from ice that had remained at the pore walls. After the last CT scan at 84 hours, the weight was followed for a total elapsed time of 110 hours. It remained constant during this time. We therefore conclude that the sublimation process had terminated by 84 hours.

\section{Image comparison}

Here, we demonstrate the processing of micro-CT images and the comparison between images of the original snow and the digital replica. The reference point for the comparison is a CT scan of the original snow structure of the sample before it was cast with DEP. After the samples had reached a constant weight, and therefore we could assume that all ice had sublimated, another CT scan was taken. 


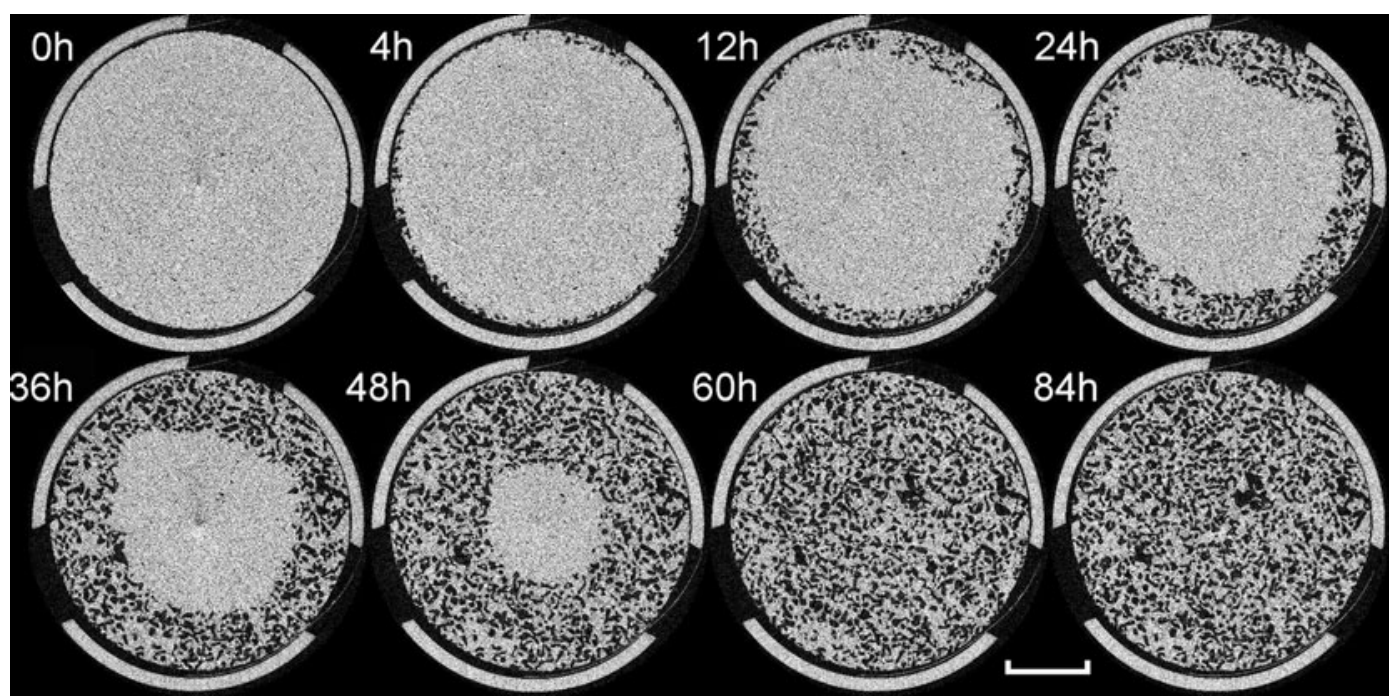

Fig. 4. Sequence of micro-CT scans that shows a cross-section of one sample. The sample was stored under vacuum without drying agent. The sublimation front propagates towards the centre of the sample. The length of the scale bar is $5 \mathrm{~mm}$.

During the casting process the DEP is expected to fill the space between the ice structures. We therefore expect that the DEP forms a 3-D negative of the original ice microstructure. Figure $5 \mathrm{a}$ and $\mathrm{b}$ show corresponding micro-CT slices of snow and DEP. Already the greyscale images of the phthalate sample show that this is the negative of the snow. Figure $5 \mathrm{c}$ and $\mathrm{d}$ show the respective segmented images. Here it becomes obvious that the DEP forms a precise negative of the snow structure.

If the black and white areas of the segmented DEP (Fig. 5d) are inverted, a digital replica similar in appearance to the snow image is achieved. This digital replica can now be compared with the segmented snow image. A visual comparison shows that the digital replica reproduces the original snow structure very well. This replica can be used for visualization purposes, for the determination of structuredescribing parameters, or as input data for numerical simulations in the same way as if the snow had been measured directly in the micro-CT instrument. We assess the accuracy of the replication below.

The absolute difference between a slice of the original snow structure and its digital replica is shown in Figure 5e and $f$. The differences form thin rims around the structural parts that are mostly only 1 voxel wide. The differences were analysed in 16 sub-volumes $(200 \times 200 \times 200$ voxels each $)$ taken from two different samples. Differences with a positive sign were slightly more frequent $(3.5 \pm 0.8 \%$ of all voxels of the specific sub-volume) than were differences with a negative sign $(2.9 \pm 0.8 \%)$. However, the difference between positive and negative differences is small $(0.6 \pm 0.8 \%)$. Positive differences are usually more frequent on one side of a structure, whereas negative differences are usually mainly found on the opposite side of a structural element.

The 3-D image was subjected to one erosion cycle with the smallest possible structuring element. This structuring element had the origin in the centre and a support of one. The shape was chosen to approximate a sphere. It thus consisted of 7 voxels and had a cross-like shape. No structures remained after this erosion, showing that all structures were $<1$ voxel, and that the difference was really caused by a sub-voxel length shift of images.
Visual inspection of the two images tells us that all the major structures are well represented in the replica. Some fine structural details, such as small asperities or thin connections between grains, may not always be replicated accurately. However, connecting elements missing on one slice are often found on an adjacent slice. Thus, these should be considered as small shifts between images rather than as missing connections.

\section{Structural parameters}

In many cases, we are more interested in parameters describing the microstructure than in the structural details. We compared the structural parameters computed from the digital replica with those of the original structure. The density, SSA and thickness distributions were chosen. On average, sample A (sample B) has a density of $361 \pm 18 \mathrm{~kg} \mathrm{~m}^{-3}$ $\left(444 \pm 6 \mathrm{~kg} \mathrm{~m}^{-3}\right)$ and an SSA of $1.88 \times 10^{4} \pm 0.04 \times 10^{4} \mathrm{~m}^{-1}$ $\left(1.49 \times 10^{4} \pm 0.02 \times 10^{4} \mathrm{~m}^{-1}\right)$. The average ice thickness is $1.68 \times 10^{-4} \pm 0.04 \times 10^{-4} \mathrm{~m}\left(2.00 \times 10^{-4} \pm 0.02 \times 10^{-4} \mathrm{~m}\right)$ and the average pore thickness $1.98 \times 10^{-4} \pm 0.15 \times 10^{-4} \mathrm{~m}$ $\left(1.96 \times 10^{-4} \pm 0.05 \times 10^{-4} \mathrm{~m}\right)$. Comparing structural parameters of the digital replica to those of the original snow structure generally shows relative errors $<5 \%$. In some cases, the relative errors are up to about $10 \%$ (Table 1).

For the two parameters of ice thickness and pore thickness, not only the average value per volume of interest (VOI) was calculated, but also the frequency distribution of the parameters (Fig. 6). The curves show the frequency of ice parts, or of pores, with a certain thickness. In Figure 6, the bold solid lines show the distribution for the original snow structures, whereas the fine dotted lines are the differences between the snow and the digital replica. The frequency distributions for the digital replicas match very well the distributions determined for the original snow, with differences $<2 \%$. Even the oscillations caused by the discretization of the images are often similar for both cases.

\section{Stability of DEP structure}

To test the stability of the DEP structure, one sample was stored at $-20^{\circ} \mathrm{C}$ for 2 months after the final CT scan. It was then scanned again using micro-CT to detect potential 

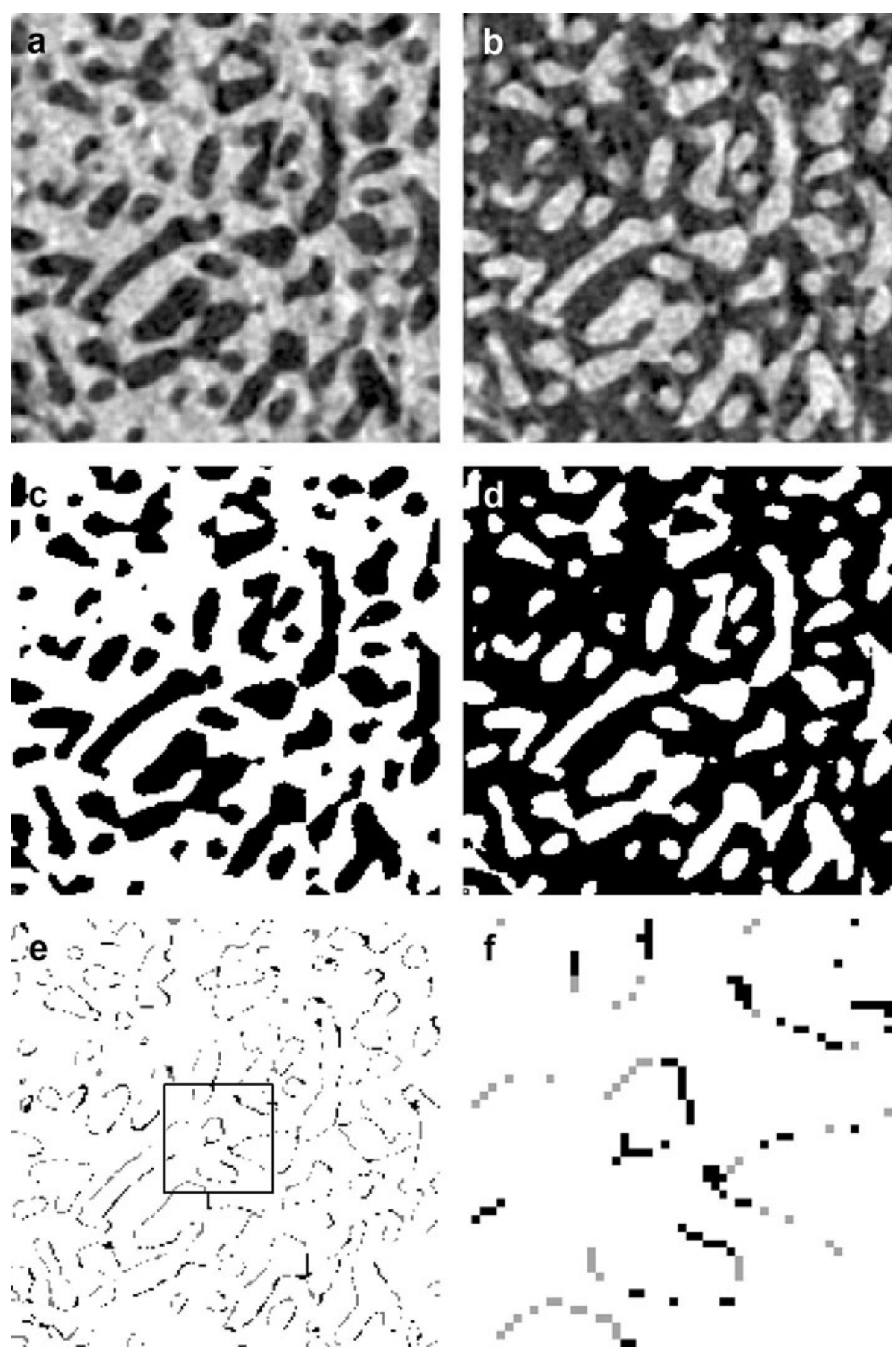

Fig. 5. The raw micro-CT images show that the DEP replica (b) is the negative of the original snow structure (a). The middle row shows the segmented images of snow (c) and replica (d). By direct inversion of the segmented image of the replica we get an image (not shown) that corresponds to the image of the snow structure (c). Differences between the two segmented images (e,f) are caused by small misalignments of the sample. The enlarged region $(\mathrm{f})$ shows that the differences are not more than 1 voxel thick. Positive differences are shown in grey and negative differences in black. The size of the slices is $3.6 \mathrm{~mm} \times 3.6 \mathrm{~mm}(0.9 \mathrm{~mm} \times 0.9 \mathrm{~mm}$ for the zoom). All image processing was performed in 3-D, but here a single slice is shown for clarity.

changes. The result shows a strong visual similarity between the two scans. Moreover, the relative errors in structural parameters between the scans at the two points in timeare very small (in all cases $<3 \%$ ). From this we conclude that the DEP structure does not change over the 2 month period and that the time between the vacuum treatment and the micro-CT measurement is not critical when stored as described.

The differences between two scans of the DEP structure appear similar to those observed between snow structure and phthalate structure. The differing voxels form thin, usually
1 voxel wide, rims around the structures, indicating that the difference is caused by imperfect alignment of the scans.

\section{DISCUSSION}

\section{Vacuum pressure and weight loss of samples}

The weight loss of the samples is caused by sublimation of the ice from the interpenetrating network of ice and DEP. The sublimation process is quite fast because the pressure in the vacuum desiccator is well below the vapour pressure of ice 
Table 1. Relative errors (in \%) between structural parameters of the digital replica and the original snow sample. Values for the density, the specific surface area (SSA), the average ice thickness and the average pore thickness are given for two samples: A (11 sub-volumes) and B (5 sub-volumes). Sample A (sample B) has a density of $361 \pm 18 \mathrm{~kg} \mathrm{~m}^{-3}\left(444 \pm 6 \mathrm{~kg} \mathrm{~m}^{-3}\right)$ and an SSA of $18.8 \pm 0.4 \mathrm{~mm}^{-1}\left(14.9 \pm 0.2 \mathrm{~mm}^{-1}\right)$. The average ice thickness is $0.168 \pm 0.004 \mathrm{~mm}(0.200 \pm 0.003 \mathrm{~mm})$ and the average pore thickness is $0.198 \pm 0.015 \mathrm{~mm}(0.196 \pm 0.005 \mathrm{~mm})$

\begin{tabular}{|c|c|c|c|c|c|c|c|c|c|c|c|c|c|c|c|c|}
\hline & A. 1 & A. 2 & A. 3 & A. 4 & A. 5 & A. 6 & A. 7 & A. 8 & A. 9 & A. 10 & A. 11 & B. 1 & B. 2 & B. 3 & B. 4 & B. 5 \\
\hline Density & -3.1 & -7.3 & -8.2 & -9.8 & -2.3 & 1.6 & 2.2 & -1.9 & -1.0 & 2.1 & -3.9 & 3.0 & 0.3 & 0.0 & -2.6 & 1.1 \\
\hline SSA & 4.5 & 6.7 & 6.6 & 6.9 & 3.2 & 0.7 & 0.1 & 1.9 & 1.6 & 0.4 & 3.6 & 1.4 & 2.2 & 2.8 & 4.1 & 1.5 \\
\hline Ice thickness & -4.3 & -6.0 & -5.9 & -6.3 & -3.2 & -1.2 & 0.6 & -2.1 & -1.8 & -0.8 & -2.6 & -1.2 & -2.4 & -2.1 & -3.1 & -1.7 \\
\hline Pore thickness & -0.5 & 2.5 & 2.5 & 4.8 & 0.0 & -3.3 & -3.4 & 0.4 & -0.4 & -2.2 & 1.4 & -5.9 & -3.0 & -3.3 & -0.5 & -3.4 \\
\hline
\end{tabular}

but well above that of DEP at $253 \mathrm{~K}$. The weight is reduced at an approximately constant rate. Hence, it seems that, at least for the sample size used, the diffusion of water molecules through the pore space of the sample does not limit the speed of the complete process. Rather we assume that the pumping efficiency of the vacuum pump at low pressure limits the process. The constant pressure of $\sim 5 \mathrm{~Pa}$ that is observed during the sublimation process is caused by a constant supply of sublimating water vapour and the limited intake of the pump. The mean free path of water molecules at the operating pressure is several millimetres, which may slow down pumping with the set-up used. A clear drop in the vacuum pressure can be observed once the generation of
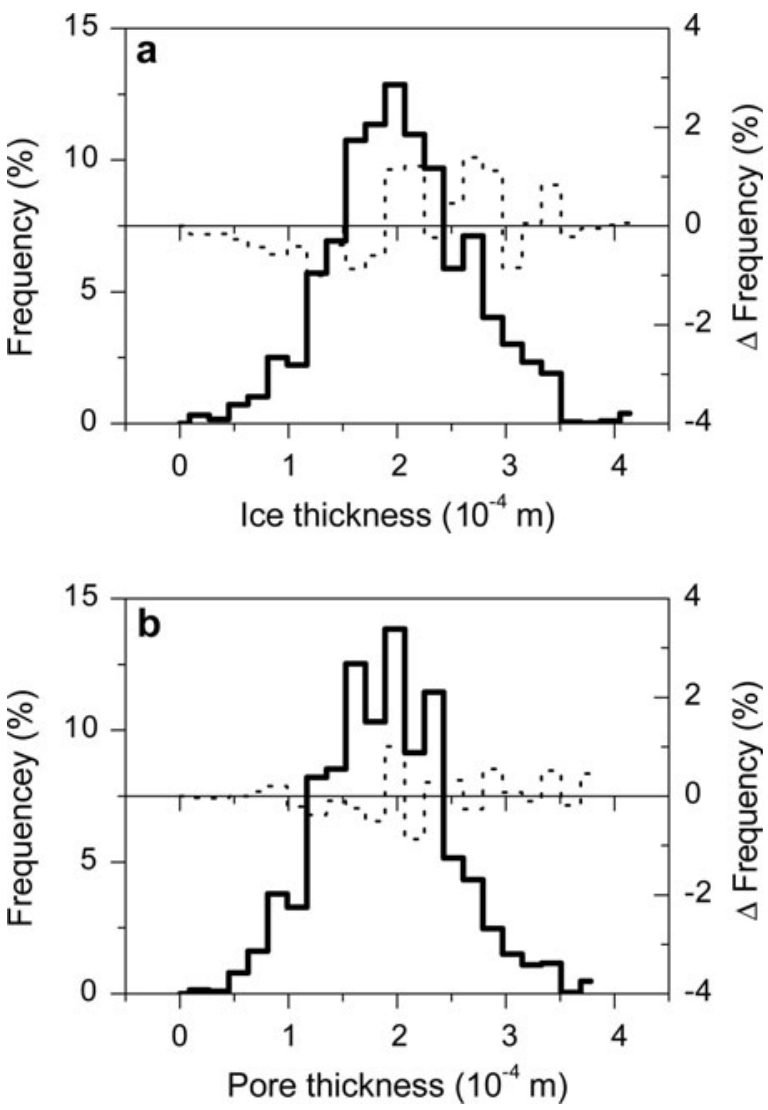

Fig. 6. Typical example of the frequency distribution of (a) ice and (b) pore thickness of subsample B.4 (Table 1) with a size of $200^{3}$ voxels, i.e. $(3.6 \mathrm{~mm})^{3}$. The distributions are calculated using the distance transform method (Hildebrand and Rüegsegger, 1997). The bold lines indicate the distributions in the original snow sample. The dotted lines show the difference between the digital replica and the original snow. water vapour decreases because the remaining ice volume becomes small. This indicates that the observed pressure plateau is caused by a constant supply of water vapour.

The use of a drying agent inside the vacuum desiccator absorbs the water vapour more efficiently than it can be pumped with the current set-up. This explains the significantly lower pressure of $\sim 0.5$ Pa measured, as well as the five-fold acceleration of the sublimation process.

\section{Image comparison}

Several possible reasons for the observed differences between the images of the original snow structure and the digital replica have to be considered: (1) the two structures have undergone physical size change due to preparation; (2) the differences are caused by measurement errors between the individual measurements; or (3) the differences are due to geometrical changes caused by the segmentation.

It is not possible to judge on the first point because there is no structure measurement independent of the micro-CT data. However, differences are small, so it can be concluded that the digital replica reproduces the snow structure. The weighing experiments showed that, on one hand, all snow mass had sublimated at the end of the experiment, while, on the other hand, the DEP sample showed no weight change even on a timescale much longer than the sublimation time. We are therefore confident that all ice can be removed from the samples without changing the phthalate structure.

Measurement errors caused by noise or mechanical inaccuracies of the instrument cannot be avoided. But as the comparison of the resulting images shows, such errors are either limited in their effect or corrected by the image processing.

Another type of measurement error occurs because the sample needs to be removed from the CT scanner between two measurements. Although there is a guidance facility to position the sample holder in the CT scanner, it is impossible to reposition the sample identically to the previous scan. There are inevitably small shifts between the images of different scans. These are taken into account during the image evaluation by searching the best matching position of the two pictures. Positioning errors were usually in the order of 1-3 voxels (i.e. $20-55 \mu \mathrm{m}$ ), with the exception of one sample where the vertical shift was 10-11 voxels (i.e. $\sim 200 \mu \mathrm{m})$. Because of the discrete nature of the digital images, the positioning errors cannot be corrected on the sub-voxel level. The differences between the digital replica and the snow structure, on the one hand, and between the digital replicas measured at two different points of time, on the other hand, are similar. Erosion showed that the differences are smaller than 1 voxel. This fact clearly 
indicates that the differences in both cases are caused by the described small positional shifts. These small errors are inevitable with the set-up used.

The third source of error can be excluded because wrong segmentation thresholds would generate errors that are either predominantly positive or predominantly negative. Since the fractions of positive and negative errors differ by only $0.6 \%$, the optimal threshold of the negative sample is correctly chosen to receive a digital replica.

\section{Structural parameters}

To rate the relative errors occurring in the structural parameters, we compare them with the coefficient of variation of parameters calculated from different sub-volumes of the snow samples. This coefficient of variation is 2 $8 \%$ for the parameters considered. In comparison, the relative errors between the original snow structure and the digital replica are of the same order.

For the structural parameters of ice thickness and pore thickness, not only are the averaged values very similar but the frequency distributions are identical. This is a further indication that the two compared structures are almost identical. The finding that even the oscillations in the frequency curves are in many cases very similar suggests that in both compared structures even the discretization errors are comparable. Taking into account the process by which the replica images were acquired and the very good optical congruence of the images of original and replica, we can conclude that the two structures are identical on a sub-voxel level.

\section{Phthalate stability}

Two CT scans were made of a sample immediately after the sublimation had terminated and after storage for 2 months at $-20^{\circ} \mathrm{C}$. Both visually and by comparing the structural parameters, only small deviations between the two measurements were found. Indeed, the difference image looks rather similar to the difference between snow and replica, the differences are not larger than 1 voxel, and no change in structural parameters was observed. We interpret these findings as an indication that the observed differences are inherent to the micro-CT measurement and image processing rather than due to real differences between the scanned objects. We conclude that the time lapse between sublimation and micro-CT scanning is not critical.

\section{Air bubbles}

It is often unavoidable that some trapped air bubbles remain in the DEP during the casting process. These are difficult to remove, especially under field conditions. In the laboratory a vacuum casting technique can be used to reduce the volume of air bubbles. However, with our method the problem can be addressed by acquiring a micro-CT scan of the cast sample. In such a scan, air bubbles can be readily identified. During the subsequent image processing, the bubble volume is added to the pore volume.

\section{CONCLUSIONS}

The fact that DEP is stable in the applied vacuum and all the ice mass can be removed from the samples, together with the accurate optical correlation and the small relative errors between the structural parameters, indicates that the original snow structure is very well replicated by the digital phthalate replica.

The sublimation method has been tested for several cast samples. This new type of 3-D reconstruction eliminates the many difficulties that occur in image processing of serial sections, especially the problem of phthalate recrystallization. The method presented here allows imaging of much larger volumes of fragile low-density snow samples and especially of layered samples. The measured data are used for visualization and quantification of snow structures, but also as input models for numerical simulations.

\section{ACKNOWLEDGEMENTS}

We thank R. Frei, D. Schmid and S. Steiner for help in the laboratory. The image-matching IDL program of T. Theile facilitated the work on this project. The valuable comments of the anonymous reviewers did much to improve the manuscript.

\section{REFERENCES}

Brzoska, J.-B. and 7 others. 1999. 3D visualization of snow samples by microtomography at low temperature. ESRF Newsl. 32, 22-23.

Coléou, C., B. Lesaffre, J.B. Brzoska, W. Ludwig and E. Boller. 2001. Three-dimensional snow images by $\mathrm{X}$-ray microtomography. Ann. Glaciol., 32, 75-81.

Flin, F., J.B. Brzoska, B. Lesaffre, C. Coléou and R.A. Pieritz. 2003. Full three-dimensional modelling of curvature-dependent snow metamorphism: first results and comparison with experimental tomographic data. J. Phys. D, 36(10A), A49-A54.

Good, W. 1987. Thin sections, serial cuts and 3-D analysis of snow. IAHS Publ. 162 (Symposium at Davos 1986 - Avalanche Formation, Movement and Effects), 35-48.

Greene, E.M. 2007. The thermophysical and microstructural effects of an artificial ice layer in natural snow under kinetic growth metamorphism. (PhD thesis, Colorado State University.)

Hildebrand, T. and P. Rüegsegger. 1997. A new method for the model independent assessment of thickness in threedimensional images. J. Microsc., 185, 67-75.

Hildebrand, T., A. Laib, R. Müller, J. Dequeker and P. Rüegsegger. 1999. Direct 3-D morphometric analysis of human cancellous bone: microstructural data from spine, iliac crest and calcaneus. J. Bone Mineral Res., 14(7), 1167-1174.

Howe, P.D., C. Melber, J. Kielhorn and I. Mangelsdorf. 2001. Chlorinated naphthalenes. Geneva, World Health Organisation. (Concise International Chemical Assessment Document 34.)

Kaempfer, T.U. and M. Schneebeli. 2007. Observation of isothermal metamorphism of new snow and interpretation as a sintering process. J. Geophys. Res., 112(D24), D24101. (10.1029/2007JD009047.)

Kaempfer, T.U., M. Schneebeli and S.A. Sokratov. 2005. A microstructural approach to model heat transfer in snow. Geophys. Res. Lett., 32(21), L21503. (10.1029/2005GL023873.)

Kerbrat, M., B. Pinzer, T. Huthwelker, H.W. Gäggeler, M. Ammann and M. Schneebeli. 2008. Measuring the specific surface area of snow with X-ray tomography and gas adsorption: comparison and implications for surface smoothness. Atmos. Chem. Phys., 8(5), 1261-1275.

Matzl, M. and M. Schneebeli. 2006. Measuring specific surface area of snow by near-infrared photography. J. Glaciol., 52(179), 558-564.

Perla, R.I., J. Dozier and R.E. Davis. 1986. Preparation of serial sections in dry snow specimens. J. Microsc., 141, 111-114.

Pielmeier, C. and M. Schneebeli. 2003. Stratigraphy and changes in hardness of snow measured by hand, ramsonde and snow micro 
penetrometer; a comparison with planar sections. Cold Reg. Sci. Technol., 37(3), 393-405.

Roháč, V. and 6 others. 2004. Vapour pressure of diethyl phthalate. J. Chem. Thermodyn., 36(11), 929-937.

Schneebeli, M. 2001. Three-dimensional snow: what snow really looks like. In ISSW 2000. Proceedings of the International Snow Science Workshop, 1-6 October 2000, Big Sky, Montana. Bozeman, MT, American Avalanche Association, 407-408.

Schneebeli, M. 2004. Numerical simulation of elastic stress in the microstructure of snow. Ann. Glaciol., 38, 339-342.

Schneebeli, M. and S.A. Sokratov. 2004. Tomography of temperature gradient metamorphism of snow and associated changes in heat conductivity. Hydrol. Process., 18(18), 3655-3665.
Sekizawa, J. and S. Dobson. 2003. Diethyl phthalate. Geneva, World Health Organisation. (Concise International Chemical Assessment Document 52.)

Stock, S.R. 2008. Recent advances in X-ray microtomography applied to materials. Int. Mater. Rev., 53(3), 129-181.

Wagner, W., A. Saul and A. Pruss. 1994. International equations for the pressure along the melting and along the sublimation curve of ordinary water substance. J. Phys. Chem. Ref. Data, 23(3), 515-527.

Yoo, T.S., G.D. Stetten and B. Lorenson. 2004. Basic image processing and linear operations. In Yoo, T.S., ed. Insight into images: principles and practice for segmentation, registration, and image analysis. Wellesley, MA, AK Peters, 19-45.

MS received 17 September 2008 and accepted in revised form 2 January 2009 